This study aimed to compile all the relevant studies of patients presenting with pericardial tamponade before or after diagnosis of lymphoma, describe the clinical presentations of patients with lymphoma and cardiac tamponade, and assess the difference in overall survival based on the timing of cardiac tamponade diagnosis.

A comprehensive search strategy was conducted in the following databases: PubMed and Cochrane Library, using the following keywords: Lymphoma AND Cardiac Tamponade. The criteria for eligibility included cases with a confirmed diagnosis of lymphoma and cardiac tamponade, human studies, and publications in English language. The statistical analysis was performed using IBM Statistical Package for Social Sciences (SPSS) version 20. We included 48 research articles $(n=52$ cases) with adequate reporting of measured outcomes. The median age of the patients was 52 (9-94) years. Only 6 patients were noted to have primary cardiac lymphoma, while the majority of cases were considered to have secondary cardiac lymphoma (88.5\%). According to the data on the type of lymphoma reported through cytology and immunohistochemistry, 49 patients were diagnosed with non-Hodgkin lymphoma, and of these cases the most common subtype was large B-cell lymphoma (42.9\%). Overall, the average duration of illness was $14 \pm 23$ days. A total of 13 patients had distant heart sounds, 12 cases were noted to be hypotensive, and 13 subjects were found to have increased jugular venous pressure.

Our retrospective study demonstrated that most patients presented with pericardial tamponade after lymphoma diagnosis, and those were mostly secondary cardiac lymphoma of the non-Hodgkin type with large B-cell as the most common subtype. Dyspnoea, oedema, and constitutional symptoms were the most common presenting signs. The median overall survival of patients with lymphoma and cardiac tamponade is 4 months, with no significant difference in mortality in the presentation timing before and after the diagnosis of lymphoma.

Key words: lymphoma, pericardial effusion, cardiac tamponade, systematic review.

Contemp Oncol (Pozn) 2021; 25 (1): 57-63 DOI:https://doi.org/10.5114/wo.2021.103828

\section{Case study-based systematic review of literature on lymphoma- associated cardiac tamponade}

\author{
Mohammad Abrar Shareef ${ }^{1}$, Abdulaziz M. Eshaq ${ }^{2}$, Rasha Alshawaf ${ }^{3}$, \\ Emad Alharthi ${ }^{4}$, Arwa A. Al Muslat ${ }^{2}$, Reema AbuDawas ${ }^{2}$, Abdulhadi A. AlAmodi ${ }^{5}$
}

${ }^{1}$ Department of Internal Medicine, Sebasticook Valley Hospital, Pittsfield, Maine, USA ${ }^{2}$ College of Medicine, Alfiasal University Riyadh, Saudi Arabia

${ }^{3}$ Internal Medicine Department, Security Forces Hospital Program Riyadh, Saudi Arabia ${ }^{4}$ Internal Medicine Department, Prince Sultan Military Medical City Riyadh, Saudi Arabia ${ }^{5}$ Department of Epidemiology and Biostatistics, School of Public Health, College of Health Sciences, Jackson State University, Jackson, Mississippi, USA

\section{Introduction}

Lymphoma is a haematological malignancy arising from the clonal proliferation of lymphocytes (B, T, or NK cells) at different maturation stages. It often presents with night sweats, fatigue, enlarged painless lymph nodes, and weight loss, and it is categorized into 2 large subgroups: Hodgkin (10\%) and non-Hodgkin lymphoma (90\%) [1, 2]. Diagnosis of lymphoma is confirmed by tissue biopsy and further evaluated by PET/CT scans to measure uptake of radiolabelled fluorodeoxyglucose to assess the biological activity of lymphoma in different sites. Pericardial involvement in systemic malignancies is common and is seen in around $1-20 \%$ of autopsies in cancer patients $[3,4]$. Pericardial involvement includes various degrees of pathologies such as pericarditis, pericardial effusion, pericardial tamponade, and constrictive pericarditis.

Pericardial effusion is the accumulation of fluid in the pericardial sac. Normally, there are between 10 and $50 \mathrm{ml}$ of pericardial fluid produced by ultrafiltration of plasma from the epicardial capillaries and effusion of interstitial fluid from the underlying myocardium. Large symptomatic pericardial effusions are often the initial clinical presentation of many systemic malignancies [5]. Symptomatic effusion often presents with dyspnoea, tachycardia, and echocardiographic features of right heart impairment $[5,6]$. Pericardial effusion stems mainly from blockage of the lymphatic and venous drainage of the pericardium by malignant seeds or via compression from neighbouring enlarged lymph nodes. Importantly, pericardial effusions in cancer patients can also be triggered by chemotherapy and radiotherapy [7-11]. Other causes of effusion, such as infections and autoimmune diseases, are also seen in these patients.

Cardiac tamponade is an accumulation of pericardial fluid with compression of all cardiac chambers due to increased pericardial pressure [12, 13]. Under normal conditions, the pericardium can accommodate physiological changes in cardiac volume. However, after its reserve volume is exceeded, or in cases of acute effusions, impairment of the underlying haemodynamics of the left and right heart chambers occurs. Venous return and (subsequently) cardiac output are compromised.

Multiple case reports have been published including cases of Hodgkin and non-Hodgkin Lymphoma patients presenting with pericardial effusion [14-18]. However, pericardial effusion leading to cardiac tamponade as an initial presentation of lymphoma is rare. Moreover, the chronological relationship between lymphoma and pericardial tamponade is not very well understood. In this review, we aimed to compile all the relevant studies of patients presenting with pericardial tamponade before or after diagnosis 
of lymphoma, describe the clinical presentations of patients with lymphoma and cardiac tamponade, and assess the difference in overall survival based on the timing of cardiac tamponade diagnosis.

\section{Material and methods}

\section{Literature search}

A comprehensive search strategy was conducted in the following databases: PubMed and Cochrane Library, using the following keywords: Lymphoma AND Cardiac Tamponade. The search was performed on 10 January 2020, and there was no restriction identified in terms of the publication period. The literature search was done in compliance with PRISMA (Preferred Reporting Item for Systematic Reviews and Meta-Analysis). All clinical studies were then extracted using the title and abstract of each study. The criteria for eligibility included cases with a confirmed diagnosis of lymphoma and cardiac tamponade, human studies, and publication in English language. The exclusion criteria entailed unconfirmed diagnosis of lymphoma, presence of pericardial effusion without cardiac tamponade, and lack of full texts or measured outcomes.

\section{Quality assessment}

The first 3 authors independently examined the retrieved the search items and ensured there were no major discrepancies or duplicates. The quality assessment tool that was developed by Murad et al. was utilized to evaluate the quality of each case study. This tool assesses each study in 4 domains: selection, ascertainment, causality, and reporting. Each domain carries 1 point, and hence, each case is scored out of 4 . Scores of 1 and 2 represent poor quality, 3 is considered fair, and 4 indicates good quality. Questions about drug events were removed due to the lack of their application in the present study.

\section{Data extraction}

The following information was extracted from each study: age, gender, clinical presentation and duration of

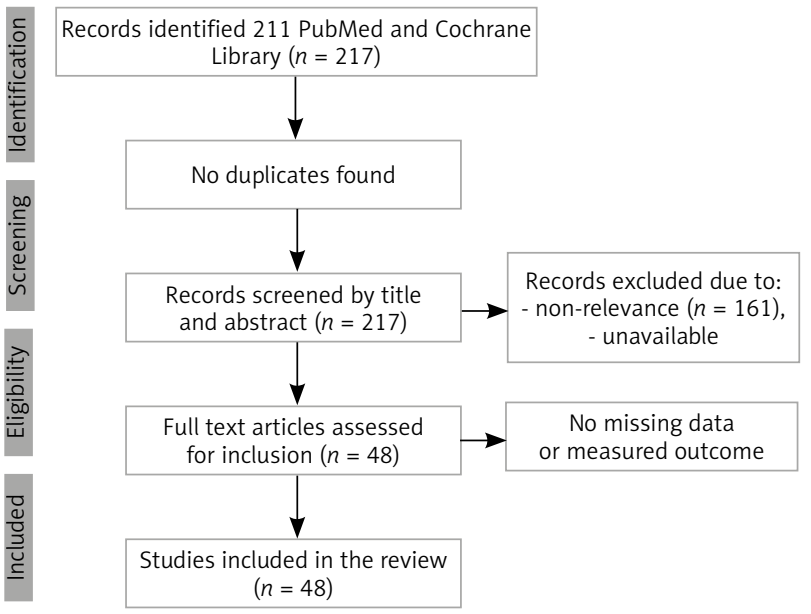

Fig. 1. Literature search strategy using PRISMA guidelines illness, the timing of cardiac tamponade diagnosis (whether before or after diagnosis of lymphoma), and associated comorbid conditions or infections, in addition to the presence of any other cancer. Information regarding diagnostic studies, management strategies, and prognosis was also gathered from these reports.

\section{Statistical analysis}

The descriptive statistics were performed by addressing the number and percentage of cases for each measured categorical variable. Data were reported for continuous variables as mean followed by standard deviation, or median followed by a range or $95 \%$ confidence interval. Survival analysis was processed using Kaplan-Meyer curves, and the results were reported in graphs, in addition to median survival and its 95\% confidence interval. The logrank test was used to examine the difference in median survivals of 2 independent groups. A $p$-value of less than 0.05 was considered statistically significant. The statistical analysis was processed using IBM Statistical Package for Social Sciences (SPSS) version 20.

\section{Results}

The literature search of identified terms in PubMed and Cochrane Library yielded a total of 217 research articles, and no systematic review was found. After reviewing each study's title and abstract, 161 studies were excluded due to non-relevance, and 7 other studies were also removed due to the unavailability of abstracts and full texts. The final number of eligible studies for analysis was 48 research articles with adequate reporting of measured outcomes. These studies consisted of case reports and case series and described a total of 52 cases (Figure 1).

A quality assessment tool was utilised to examine each enrolled case study's quality, and, when applying this method, 23 cases were identified as good quality, 19 cases were marked as fair quality, while 10 cases were reported to be of poor quality. The median age of the patients was 52 years, ranging between 9 and 94 years. Around $73 \%$ of cases were reported in male patients, and 14 cases were described in female patients. Most of the patients did not report any comorbid condition (75\%). However, 5 patients had chronic arrhythmias: 3 had a history of third-degree atrioventricular block and had a pacemaker in place, while 2 patients had chronic atrial fibrillation. Moreover, 3 patients were diagnosed with other cancers in the past, including hepatocellular carcinoma, basal cell cancer, and squamous cell carcinoma of the vocal cords (Table 1).

Most of the patients were diagnosed with lymphoma prior to hospital presentation (80.8\%). Only 6 patients were noted to have primary cardiac lymphoma, while the majority of cases were considered to have secondary cardiac lymphoma (88.5\%). Upon reviewing the type of lymphoma through cytology and immunohistochemistry, 49 patients were diagnosed with non-Hodgkin lymphoma, and of these cases, the most common subtype was large B-cell lymphoma (42.9\%).

A large majority of patients presented to the hospital complaining of increased shortness of breath (94.2\%), and 
around $35 \%$ of the cases presented with constitutional symptoms, including fever, chills, night sweat, myalgia, or fatigue. Overall, the average duration of illness was 14 days with a standard deviation of 23 days. A total of 13 patients had distant heart sounds, 12 cases were noted to be hypotensive, and 13 subjects were found to have increased jugular venous pressure (Figure 2).

About $65.4 \%$ of cardiac tamponade patients in the present study underwent a transthoracic echocardiogram, and four of them had a subsequent transoesophageal echocardiogram. The common echocardiogram finding was pericardial effusion (82.4\%) followed by right atrial mass or collapse (32.4\%). Furthermore, right ventricular diastolic collapse was noted in 10 case studies, while left ventricular collapse was demonstrated in 3 cases. However, there was no reporting of inferior vena cava dilation. Chest $X$-ray, on the other hand, was obtained in 30 cases, and chest computed tomography was performed in around 54\% of the cases (Table 2). The most common radiographic finding was cardiomegaly or pericardial effusion, with or without pleural effusion (61.5\%). Moreover, an electrocardiogram was performed in 15 patients, which revealed sinus tachycardia in half of the tested cases. Low-voltage QRS complexes were depicted in 3 cases, but there was no reporting of electrical alternans. In terms of laboratory workup, elevated lactate dehydrogenase (LDH) was found in $23 \%$ of the cases, and leukocytosis was noted in 6 patients. Cytology and immunohistochemistry results varied depending on the type of lymphoma.

The most common therapeutic option that was offered to these patients for the management of cardiac tamponade was pericardiocentesis (63.5\%), followed by the pericardial window (15.4\%). The amount of aspirated pericardial fluid was reported in 14 studies with an average of $862 \pm 930 \mathrm{ml}$, and all fluid analyses revealed exudative fluid. Some patients had concomitant pleural effusion, and these cases underwent thoracentesis, which showed similar fluid analysis results (9.6\%). Meanwhile, 34 patients were administered chemotherapy for the management of lymphoma (65.4\%), and 7 patients underwent surgical resection (13.5\%).

The median overall survival of patients with lymphoma and cardiac tamponade is 4 months, with a $95 \%$ confidence interval of 0 to 12 months (Figure 3 ). As stated earlier, compared to patients who were known to have lymphoma prior to presenting with cardiac tamponade, only 10 patients were diagnosed with lymphoma after diagnosis of cardiac tamponade. To explore if this was a potential influencing factor on the overall survival curve, the logrank test was utilized. The test demonstrated no significant difference between median survivals of the 2 groups $(p=0.24)$ (Figure 4). Due to the small sample size of patients who had lymphoma diagnosis after cardiac tamponade $(n=10)$ and thus fewer deaths, the median survival could not be computed in that group.

\section{Discussion}

The incidence of haematological malignancies is on the rise in Africa. The number of new lymphoma cases is expect-
Table 1. Characteristics of patients in the study $(n=52)$

\begin{tabular}{|lc|}
\hline Characteristic & Number of patients (\%) \\
Age (years), median (range) & $52(9-94)$ \\
Gender & \\
\hline Male & $38(73.1)$ \\
\hline Female & $14(26.9)$ \\
\hline Comorbid conditions* & \\
\hline One & $4(7.7)$ \\
\hline Two & $3(5.8)$ \\
\hline Three & $2(3.8)$ \\
\hline More than three & $4(7.7)$ \\
\hline Presence of other cancers & $3(5.8)$ \\
\hline Arrhythmia & \\
\hline Atrial fibrillation & $2(3.9)$ \\
\hline Third degree atrioventricular block & $3(5.8)$ \\
\hline Associated infections & \\
\hline Human immunodeficiency virus & $3(5.8)$ \\
\hline Human herpesvirus 8 & $3(5.8)$ \\
\hline Epstein-Barr virus & $2(3.9)$ \\
\hline Tuberculosis & $2(3.9)$ \\
\hline Infectious mononucleosis & $1(1.9)$ \\
\hline Hepatitis B virus & $1(1.9)$ \\
\hline Hepatitis C virus & $1(1.9)$ \\
\hline Type of cardiac lymphoma & \\
\hline Primary cardiac lymphoma & $6(11.5)$ \\
\hline Becondary cardiac lymphoma & $46(88.5)$ \\
\hline After lymphoma diagnosis & $42(80.8)$ \\
\hline Pomore lymphoma diagnosis & $10(19.2)$ \\
\hline
\end{tabular}

${ }^{*}$ Comorbid conditions - abdominal aortic aneurysm, asthma, Barret's oesophagus, chronic renal disease, congestive heart failure, coronary artery disease, diabetes mellitus, haemolytic anaemia, hyperlipidaemia, hypertension, hypothyroidism, obesity, obstructive sleep apnoea, peripheral neuropathy, pulmonary fibrosis

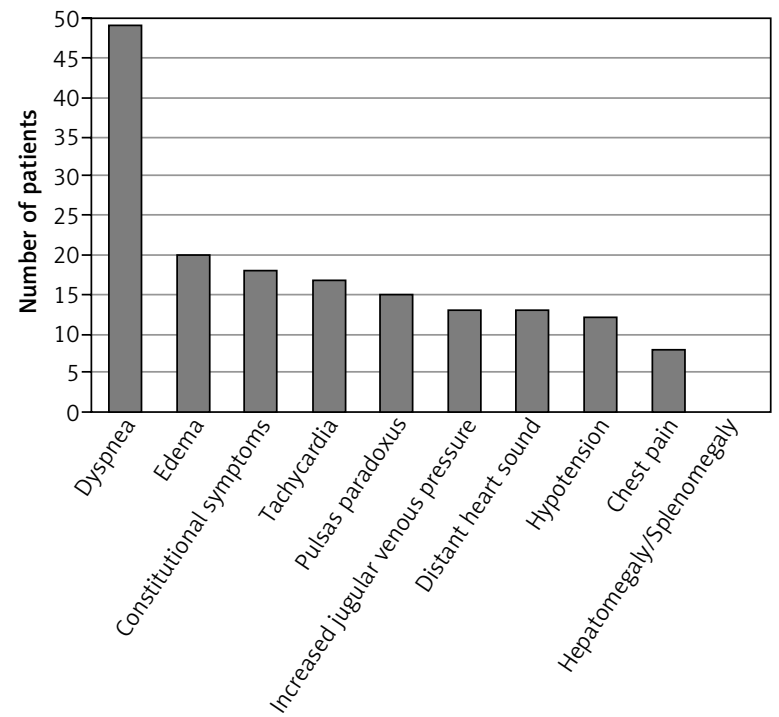

Fig. 2. Clinical presentations of patients with lymphoma and cardiac tamponade 
Table 2. Diagnosis and management of patients with lymphoma and cardiac tamponade

\begin{tabular}{|lc|}
\hline Diagnostic and therapeutic modalities & Number of patients (\%) \\
\hline Diagnostic workups & \\
\hline CXR & $30(57.7)$ \\
\hline CT chest & $28(53.8)$ \\
EKG & $15(28.8)$ \\
\hline Echocardiogram & $34(65.4)$ \\
\hline Cardiac MRI & $6(11.5)$ \\
\hline Cytology & $21(40.4)$ \\
\hline Immunohistochemistry & $28(53.8)$ \\
\hline Management strategies & \\
\hline Chemotherapy & $34(65.4)$ \\
\hline Pericardiocentesis & $33(63.5)$ \\
\hline Pericardial window & $8(15.4)$ \\
\hline Surgical resection & $7(13.5)$ \\
\hline Thoracentesis & $5(9.6)$ \\
\hline Radiation & $5(9.6)$ \\
\hline Bone marrow transplant & $4(7.7)$ \\
\hline Steroids & $2(3.8)$ \\
\hline Sternotomy with no resection & $2(3.8)$ \\
\hline Thoracotomy & $1(1.9)$ \\
CXR - chest X-ray, CT-computed tomography, EKG - electrocardiogram, \\
MRI - magnetic resonance imaging
\end{tabular}

ed to nearly double over the next 2 decades, from approximately 25000 in 2012 to more than 48000 by 2035 [19]. A large part of this is because of the human immunodeficiency virus (HIV) epidemic, as well as population growth and aging. Lymphoma diagnosis and classification are heavily reliant on flow cytometry, immunohistochemistry, and cytogenetics, which are expensive and largely unavailable in most African countries, especially sub-Saharan Africa. Likewise, intensive cytotoxic chemotherapy regimens are often not tolerable in settings with limited

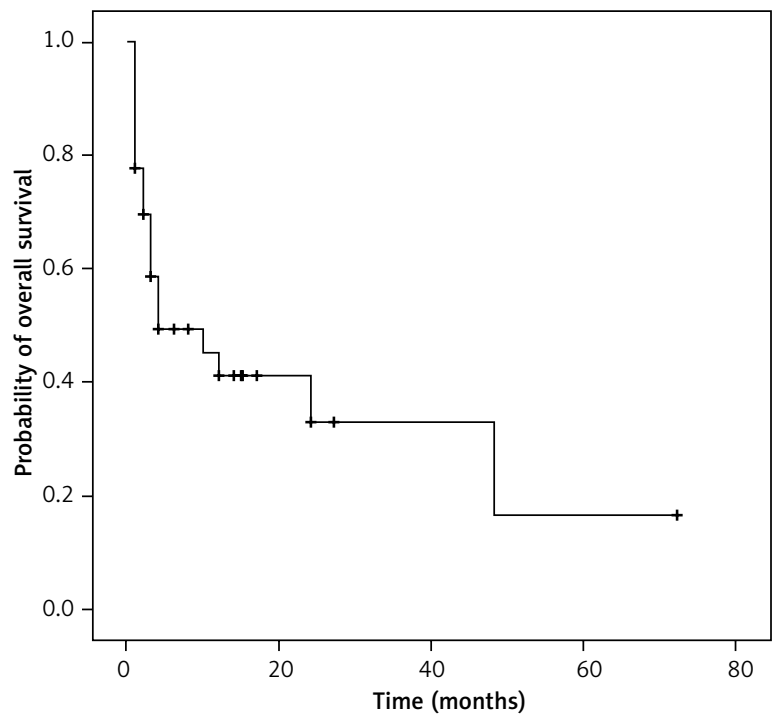

Fig. 3. Overall survival of lymphoma patients with cardiac tamponade supportive care. Delay in diagnosis and treatment of lymphoma leads to presentation with late symptoms of metastasis. It has been suggested that one of the early signs of metastasis is effusions in endothelial-lined sacs, such as pleural and pericardial effusions [20, 21].

Cardiac tamponade is a pericardial effusion in which the pressure from the accumulating fluid equalizes with intracardiac pressures, with subsequent impairment of ventricular filling and decreased cardiac output [12, 13]. The pericardium's involvement is often the first manifestation of malignancy and may necessitate a further evaluation of occult cancer. This should be reserved for patients who present with persistent acute or recurrent pericarditis, unresponsiveness to anti-inflammatory therapy, concern for malignancy (e.g. weight loss), and new large pericardial effusion or cardiac tamponade.

Occult malignancies in patients initially presenting with a large pericardial effusion or cardiac tamponade are common. In a case series of 57 patients with a new large effusion, pericardial biopsy was found about $25 \%$ of undiagnosed malignancy patients [22]. In another report of 450 patients with acute pericardial disease, $7 \%$ of patients had a neoplastic cause with significant association with cardiac tamponade at presentation and lack of response to anti-inflammatory drugs [23]. In our review of 52 cases, most patients (80.8\%) were diagnosed with lymphoma before hospital presentation and were considered to have secondary cardiac lymphoma (88.5\%). Non-Hodgkin B-cell lymphoma was the most common type (42.9\%).

In our review, patients mostly presented with dyspnoea (94.2\%), oedema (35\%), and constitutional symptoms (35\%). Clinical presentation of cardiac tamponade usually depends upon how quickly fluid accumulates inside the pericardial sac [24]. Acute cardiac tamponade happens when there is a small but sudden life-threatening accumulation of fluid. In this scenario, the parietal pericardium fails to accommodate the increase in volume, and an acute rise in pericardial pressure ensues [12]. Subacute or haemodynamically insignificant pericardial effu-

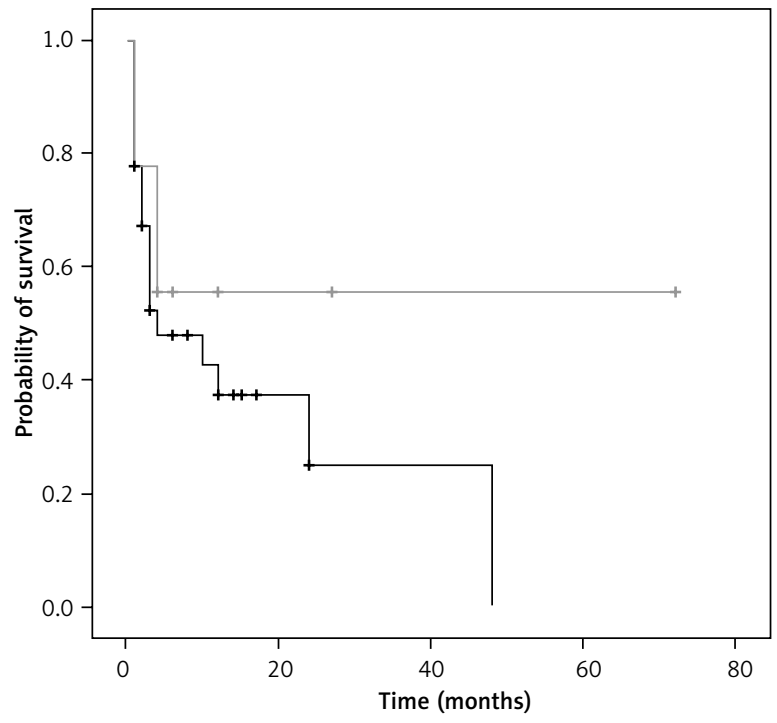

Fig. 4. Difference in overall survival based on timing of cardiac tamponade diagnosis ( $p=0.24$ using log-rank test) 
sion presents with no specific symptoms, but patients may still have symptoms related to the underlying cause (e.g. fever due to pericarditis). Subacute cardiac tamponade is a less dramatic process in which fluid accumulates slowly, and thus the pericardium can accommodate the growing volume [25]. Patients may be asymptomatic or complain of dyspnoea or fatigue. Cardiac tamponade is usually subacute in the setting of malignancy.

The initial workup for pericardial effusion includes an ECG, chest radiograph, and echocardiogram, with or without cross-sectional imaging of the chest as part of staging or restaging of malignancy [26]. Electrocardiography shows decreased QRS voltage and, occasionally, electrical alternans. Chest radiography (CXR) shows an enlarged cardiac silhouette with clear lung fields. CXRs are useful for detecting a concomitant pleural effusion. Echocardiography is warranted on suspicion of pericardial disease. It is necessary to detect the presence and estimate the pericardial effusion quantity and evaluate its haemodynamic impact on the heart. In the data from our study, about $65.4 \%$ of cardiac tamponade underwent a transthoracic echocardiogram, with the most common finding being pericardial effusion (82.4\%) followed by right atrial mass or collapse (32.4\%).

Determination of signs or symptoms suspicious for cardiac tamponade is critical in the case of effusion findings on echocardiography. Urgent pericardial drainage is recommended in the case of tamponade [27]. The most common therapeutic option that was offered to patients in our study was pericardiocentesis (63.5\%), followed by pericardial window (15.4\%). If there is no evidence of tamponade, pericardial fluid sampling or biopsy may still be needed to document the potential spread of malignant seeds to the pericardium. Cross-sectional imaging may help assess pericardial involvement and is necessary for staging lymphoma [28, 29].

Pericardiocentesis with cytologic and flow cytometric examination of the pericardial fluid is important if haemorrhagic effusion is present and if there is any reason to suspect malignancies [30]. The cytology's sensitivity for diagnosing a malignant effusion is about $67 \%$ in lymphoma [31, 32]. Flow cytometry of pericardial fluid is critical in lymphoma patients $[33,34]$. Pericardial biopsy or pericardioscopy may be required if cytology is negative and there is still high suspicion of malignancy [28, 29]. Cardiac catheterization can assess the haemodynamics of the right and left heart and the impact of pericardial disease upon cardiac filling and performance, particularly if echocardiography is inconclusive.

In terms of pericardial effusion therapeutic intervention, prolonged survival is the main target. Most patients presenting with a malignant pericardial effusion have a short life expectancy (median 2-4 months) [35-40]. However, the prognosis is better in haematological rather than solid tumours and in candidates for systemic chemotherapy [41, 42].

Generally, conservative observation is enough for asymptomatic or minimally symptomatic pericardial effusions. On the other hand, urgent fluid removal is warranted in highly symptomatic patients or those with evidence of haemodynamic compromise (tamponade) [5]. This is usually accomplished by percutaneous pericardiocentesis under echocardiographic guidance or by a pericardial window. Pericardiocentesis is an effective and rapid symptom in these patients, even if clinical or echocardiographic signs of cardiac tamponade persist.

Although pericardiocentesis initially relieves symptoms and improves haemodynamics, fluid accumulates in as many as $60 \%$ of cases $[39,43]$. Recurrent accumulation can be managed by catheter drainage and by creating a permanent pericardial window allowing drainage of fluid into the pleural or peritoneal cavity. In multiple case series, catheter drainage relieved tamponade in $70-90 \%$ of cases [44-46]. In a case series, recurrent fluid accumulation was recorded in $30 \%$ of cases in 3 months of follow-up, while another series recorded a $13 \%$ rate of recurrent effusion with 1 year of follow-up [40, 47]. In a recent systematic review of 31 nonrandomized studies, any intervention other than isolated pericardiocentesis (38.3\% recurrence rate) was associated with significantly lower recurrence rates. Pericardiocentesis with extended catheter drainage (486 patients) and balloon pericardiotomy (157 patients) showed recurrence rates of 12.1 and $10.3 \%$, respectively.

Surgical decompression of the pericardium (e.g. pericardiotomy, pericardiostomy, and window pericardiectomy) is performed using various surgical techniques (e.g. open surgery, video-assisted thoracoscopy). While no randomized trials have been done to compare surgical and percutaneous drainage of pericardial effusions, retrospective studies demonstrated immediate haemodynamic benefit in almost all patients with minimal post-procedural morbidity and mortality [31, 40, 48, 49]. In the data reviewed in our study, only 7 patients required surgical resection (13.5\%). A systematic review including data from 59 nonrandomised studies suggested a higher likelihood of successful control of effusion with surgical intervention as compared to a percutaneous approach [50]. However, the lack of randomized trials for this problem and likely selection bias in these reports limit the power of any recommendation.

Compared to patients known to have lymphoma before presenting with cardiac tamponade, only 10 patients were diagnosed with lymphoma after cardiac tamponade diagnosis. When assessing overall survival, tamponade timing either before or after lymphoma diagnosis was not a potential influencing factor.

Our study was limited by the scarcity of clinical trials, which necessitated the compiling of only case reports. Case reports are not generally representative of the population, because they usually represent outliers with no possibility of establishing cause-effect relationships and publication bias. Moreover, the low number of patients [10] in our study made it difficult to calculate the median survival of patients with lymphoma diagnosis after cardiac tamponade.

\section{Conclusions}

Our study of the published data demonstrates that most patients presented with pericardial tamponade after 
lymphoma diagnosis, and those were mostly secondary cardiac lymphoma of the non-Hodgkin type, with large B-cell as the most common subtype. Dyspnoea, oedema, and constitutional symptoms were the most common presenting signs. Most patients underwent echocardiography with subsequent pericardiocentesis. The median overall survival of patients with lymphoma and cardiac tamponade is 4 months, with no significant difference in mortality in the presentation timing before and after the diagnosis of lymphoma. Further, well-designed longitudinal studies are required to investigate the association between lymphoma and cardiac tamponade.

\section{The authors declare no conflict of interest.}

\section{References}

1. Matasar MJ, Zelenetz AD. Overview of lymphoma diagnosis and management. Radiol Clin North Am 2008; 175-198.

2. Mugnaini EN, Ghosh N. Lymphoma. Prim Care 2016; 43: 661-675.

3. Klatt EC, Heitz DR. Cardiac metastases. Cancer 1990; 65: 14561459.

4. Neoplasms metastatic to the heart: review of 3314 consecutive autopsies - PubMed.

5. Imazio M, Colopi M, De Ferrari GM. Pericardial diseases in patients with cancer: contemporary prevalence, management and outcomes. Heart 2020; 106: 569-574.

6. Ben-Horin S, Bank I, Guetta V, Livneh A. Large symptomatic pericardial effusion as the presentation of unrecognized cancer: a study in 173 consecutive patients undergoing pericardiocentesis. Medicine (Baltimore) 2006; 85: 49-53.

7. Dogan SE, Mizrak D, Alkan A, Demirkazik A. Docetaxel-induced pericardial effusion. J Oncol Pharm Pract 2017; 23: 389-391.

8. Heinzerling L, Ott PA, Hodi FS, et al. Cardiotoxicity associated with CTLA4 and PD1 blocking immunotherapy. J Immunother Cancer 2016; 4: 50.

9. Eskazan AE, Soysal T, Ongoren S, Gulturk E, Ferhanoglu B, Aydin Y. Pleural and pericardial effusions in chronic myeloid leukemia patients receiving low-dose dasatinib therapy. Haematologica 2011; 96: e5.

10. Escudier M, Cautela J, Malissen N, et al. Clinical features, mana gement, and outcomes of immune checkpoint inhibitor-related cardiotoxicity. Circulation 2017; 136: 2085-2087.

11. Chang HM, Okwuosa TM, Scarabelli T, Moudgil R, Yeh ETH. Cardiovascular complications of cancer therapy: best practices in diagnosis, prevention, and management: Part 2. J Am Coll Cardiol 2017; 70: 2552-2565.

12. Spodick DH. Acute cardiac tamponade. N Engl J Med 2003; 349: 684-690.

13. Troughton RW, Asher CR, Klein AL. Pericarditis. Lancet 2004; 363: 717-727.

14. Hajra A, Bandyopadhyay D, Layek M, Mukhopadhyay S. Cardiac tamponade as initial presentation of hodgkin lymphoma. J Clin Imaging Sci 2015; 5: 67.

15. Nagamine $\mathrm{K}$, Noda $\mathrm{H}$. Two cases of primary cardiac lymphoma presentinc with pericardial effusion and cardiac tamponade. Jpn Circ J 1990; 54: 1158-1164.

16. Almagro UA, Remeniuk E. Non-Hodgkin's lymphoma presenting as malignant pericardial effusion and cardic tamponade. Hum Pathol 1985; 16: 315-317.

17. Fincher RME. Case report: malignant pericardial effusion as the initial manifestation of malignancy. Am J Med Sci 1993; 305: 106110.

18. Wilhite DB, Quigley RL. Occult cardiac lymphoma presenting with cardiac tamponade. Texas Hear Inst J 2003; 30: 62-64.

19. Global Cancer Observatory.
20. Javed IN, Abdo T, Ahmad N, Jones KR. Metastatic pleural effusion: an unusual presentation of urothelial bladder carcinoma. Cureus 2019; 11: e4619.

21. Lv X, He J, Shen Y, Zheng H. Malignant pleural and pericardial effusions and meningeal infiltrates without other metastases in breast cancer: a case report. Mol Clin Oncol 2016; 4: 855-858.

22. Corey GR, Campbell PT, Van Trigt P, et al. Etiology of large pericardial effusions. Am J Med 1993; 95: 209-213.

23. Imazio M, Demichelis B, Parrini I, et al. Relation of acute pericardial disease to malignancy. Am J Cardiol 2005; 95: 1393-1394.

24. Hayes SN, Freeman WK, Gersh BJ. Low pressure cardiac tamponade: diagnosis facilitated by Doppler echocardiography. Br Heart J 1990; 63: 136-140.

25. Cardiac tamponade: a clinical challenge.

26. Tsang TSM, Oh JK, Seward JB. Diagnosis and management of cardiac tamponade in the era of echocardiography. Clin Cardiol 1999; 22: 446-452.

27. Kumar R, Sinha A, Lin M, et al. Complications of pericardiocentesis: a clinical synopsis. Int J Crit Illn Inj Sci 2015; 5: 206.

28. Nugue O, Millaire A, Porte H, et al. Pericardioscopy in the etiologic diagnosis of pericardial effusion in 141 consecutive patients. Circulation 1996; 94: 1635-1641.

29. Maisch B, Pankuweit S, Brilla C, et al. Intrapericardial treatment of inflammatory and neoplastic pericarditis guided by pericardioscopy and epicardial biopsy - results from a pilot study. In: Clinical Cardiology. Foundation for Advances in Medicine and Science Inc. 1999.

30. Atar S, Chiu J, Forrester JS, Siegel RJ. Bloody pericardial effusion in patients with cardiac tamponade. Is the cause cancerous, tuberculous, or latrogenic in the 1990s? Chest 1999; 116: 1564-1569.

31. Wilkes JD, Fidias P, Vaickus L, Perez RP. Malignancy related pericardial effusion. 127 cases from the roswell park cancer institute. Cancer 1995; 76: 1377-1387.

32. Porte HL, Janecki-Delebecq TJ, Finzi L, Métois DG, Millaire A, Wurtz AJ. Pericardoscopy for primary management of pericardial effusion in cancer patients. Eur J Cardiothorac Surg 1999; 16: 287-291.

33. Czader M, Ali SZ. Flow cytometry as an adjunct to cytomorphologic analysis of serous effusions. Diagn Cytopathol 2003; 29: 74-78.

34. Iqbal J, Liu T, Mapow B, et al. Importance of flow cytometric analysis of serous effusions in the diagnosis of hematopoietic neoplasms in patients with prior hematopoietic malignancies. Anal Quant Cytol Histol 2010; 32: 161-165.

35. Gross JL, Younes RN, Deheinzelin D, Diniz AL, Silva RA Da, Haddad FJ. Surgical management of symptomatic pericardial effusion in patients with solid malignancies. Ann Surg Oncol 2006; 13: 17321738.

36. Dequanter D, Lothaire P, Berghmans T, Sculier JP. Severe pericardial effusion in patients with concurrent malignancy: A retrospective analysis of prognostic factors influencing survival. Ann Surg Oncol 2008; 15: 3268-3271.

37. Maher EA, Shepherd FA, Todd TJR. Pericardial sclerosis as the primary management of malignant pericardial effusion and cardiac tamponade. J Thorac Cardiovasc Surg 1996; 112: 637-643.

38. Dosios T, Theakos N, Angouras D, Asimacopoulos P. Risk factors affecting the survival of patients with pericardial effusion submitted to subxiphoid pericardiostomy. Chest 2003; 124: 242-246.

39. Tsang TSM, Seward JB, Barnes ME, et al. Outcomes of primary and secondary treatment of pericardial effusion in patients with malignancy. Mayo Clin Proc 2000; 75: 248-253.

40. Allen KB, Faber LP, Warren WH, Shaar CJ. Pericardial effusion: Subxiphoid pericardiostomy versus percutaneous catheter drainage. Ann Thorac Surg 1999; 67: 437-440.

41. Verdier C, Martinez LO, Ferrières J, Elbaz M, Genoux A, Perret B. Targeting high-density lipoproteins: Update on a promising therapy. Arch Cardiovasc Dis 2013; 106: 601-611.

42. Hu ZG, Hu K, Li WX, Zeng FJ. Prognostic factors and nomogram for cancer-specific death in non small cell lung cancer with malignant pericardial effusion. PLoS One 2019; 14: e0217007. 
43. Laham RJ, Cohen DJ, Kuntz RE, Baim DS, Lorell BH, Simons M. Pericardial effusion in patients with cancer: Outcome with contemporary management strategies. Heart 1996; 75: 67-71.

44. Lekhakul A, Assawakawintip C, Fenstad ER, et al. Safety and outcome of percutaneous drainage of pericardial effusions in patients with cancer. Am J Cardiol 2018; 122: 1091-1094.

45. El Haddad D, Iliescu C, Yusuf SW, et al. Outcomes of cancer patients undergoing percutaneous pericardiocentesis for pericardial effusion. J Am Coll Cardiol 2015; 66: 1119-1128.

46. Virk SA, Chandrakumar D, Villanueva C, Wolfenden H, Liou K, Cao C. Systematic review of percutaneous interventions for malignant pericardial effusion. Heart 2015; 101: 1619-1626.

47. Patel N, Rafique AM, Eshaghian S, et al. Retrospective comparison of outcomes, diagnostic value, and complications of percutane ous prolonged drainage versus surgical pericardiotomy of pericardial effusion associated with malignancy. Am J Cardiol 2013; 112: $1235-1239$

48. Moores DWO, Allen KB, Faber LP, et al. Subxiphoid pericardial drainage for pericardial tamponade. J Thorac Cardiovasc Surg 1995; 109: 546-552.

49. Cullinane CA, Paz IB, Smith D, Carter N, Grannis FW. Prognostic factors in the surgical management of pericardial effusion in the patient with concurrent malignancy. Chest 2004; 125: 1328-1334.

50. Jama GM, Scarci M, Bowden J, Marciniak SJ. Palliative treatment for symptomatic malignant pericardial effusion. Interact Cardiovasc Thorac Surg 2014; 19: 1019-1026.

\section{Address for correspondence}

\section{MBBS Abdulhadi A. Alamodi}

Department of Epidemiology and Biostatistics

School of Public Health, College of Health Sciences

Jackson State University

lackson, Mississippi, USA 39213

phone: +1 7865686033

e-mail: alamoudi.aa89@gmail.com

Submitted: 5.12 .2020

Accepted: 3.01 .2021 